\title{
Aspectos genético-quantitativos da qualidade da carne em frangos
}

\author{
Quantitative-genetic aspects of broiler meat quality
}

\author{
Leila de Genova Gaya ${ }^{1}$ José Bento Sterman Ferraz ${ }^{2}$
}

\section{- REVISÃO BIBLIOGRÁFICA -}

\section{RESUMO}

O estudo dos parâmetros genéticos das características de qualidade de carne de aves permite à industria avícola se adequar às exigências da indústria processadora, aumentando sua eficiência, e melhorando a aceitação da carne de frango pelo mercado consumidor. Além disso, por meio do estudo destes parâmetros, valiosas informações sobre a caracterização do fenômeno denominado PSE, que representa a carne pálida, flácida e exsudativa, podem ser obtidas, uma vez que são escassos os estudos a esse respeito em frangos. $O$ conhecimento do comportamento genético e da relação entre os atributos da carne e outras características de interesse em frangos de corte pode favorecer o estabelecimento mais preciso e adequado das estratégias utilizadas nos programas de seleção.

Palavras-chave: frangos, melhoramento genético, parâmetros genéticos, PSE, qualidade da carne.

\section{ABSTRACT}

The study of genetic parameters of broiler meat quality traits allows the poultry industry to adjust itself to processing industry exigencies, increasing its efficiency, and to the consumers' demands, improving the acceptance of broiler meat. Moreover, through the study of these parameters, valuable information about the characterization of PSE phenomenon in broiler can be obtained, since studies about this topic are scarce. The knowledge about the genetic behavior and the relation among quality attributes and other interesting traits in broiler can support an accurate and an adequate establishment of the strategies used in breeding programs.

Key words: animal breeding, broiler, genetic parameters, meat quality, PSE.

\section{INTRODUÇÃO}

$\mathrm{Na}$ indústria de frangos de corte as mudanças de mercado são bastante comuns, o que requer uma melhoria contínua no esquema e nas ferramentas dos programas de melhoramento genético. A genética deve buscar aves compatíveis com as exigências altamente competitivas dos mercados produtivo, industrial e consumidor (CAMPOS \& PEREIRA, 1999). Até recentemente, o foco para seleção era apenas na taxa de crescimento, todavia, de acordo com PARK et al. (2002), características relacionadas à qualidade da carne vêm apresentando crescente importância, tanto para a indústria processadora como para os consumidores. Presume-se, inclusive, que a intensa seleção a favor da taxa de crescimento das aves levou a problemas relacionados à qualidade da carne destes animais (DRANSFIELD \& SOSNICKI, 1999). Desta forma, estas características passaram a ser consideradas como objeto de estudo nos programas de seleção.

Qualidade da carne

A carne utilizada em produtos processados deve possuir propriedades funcionais excelentes, com padrões de qualidade estáveis, que garantam um produto final de boa qualidade e rentabilidade (BRESSAN, 1998). Entretanto, segundo DIRINCK et al. (1996), um dos maiores desafios para a indústria de carnes é oferecer produtos macios, suculentos e com cor e sabor agradáveis.

\footnotetext{
${ }^{1}$ Departamento de Ciências Básicas, Faculdade de Zootecnia e Engenharia de Alimentos, Universidade de São Paulo (USP), Avenida Duque de Caxias Norte, 225, CP 23, CEP 13635-900, Pirassununga, São Paulo, Brasil, E-mail: genova@usp.br. Autor para correspondência.

${ }^{2}$ Departamento de Ciências Básicas, Faculdade de Zootecnia e Engenharia de Alimentos, USP, Pirassununga, SP, Brasil.
} 
Os principais atributos avaliados na carne para determinar sua qualidade são cor, capacidade de retenção de água e textura.

A cor é um dos fatores mais importantes na percepção do consumidor quanto à qualidade da carne, pois é uma característica que influencia tanto a escolha inicial do produto pelo consumidor como a aceitação no momento do consumo (FLETCHER, 1999). De acordo com OLIVO et al. (2001), a cor observada na superfície das carnes é o resultado da absorção seletiva da luz pela mioglobina e por outros importantes componentes, como as fibras musculares e suas proteínas, sendo também influenciada pela quantidade de líquido livre presente na carne. Os parâmetros utilizados na avaliação da cor da carne utilizados baseiam-se no sistema colorimétrico denominado CIELab, sigla composta pelas iniciais da comissão que estabeleceu o sistema (The Commission Internationale de L'Eclairage, em 1976) e suas escalas de cor (luminosidade, representada por $L^{*}$, teor de vermelho, representado por $a^{*}$ e teor de amarelo, representado por $\left.b^{*}\right)$.

A capacidade de retenção de água é um termo originalmente usado para descrever a capacidade do músculo e dos produtos cárneos em manter a água ligada a si (FENNEMA, 1990). A água no músculo é retida em sua maior parte intracelularmente e também entre as miofibrilas (OFFER \& KNIGHT, 1988). A capacidade de retenção de água está entre as propriedades funcionais mais importantes da carne (ANADÓN, 2002), pois influencia seu aspecto, sua palatabilidade e está diretamente relacionada às perdas de água antes e durante o cozimento (BRESSAN, 1998). A textura é outro fator bastante importante na percepção do consumidor quanto à qualidade da carne (BRESSAN, 1998). A textura da carne está intimamente relacionada à quantidade de água intramuscular e, portanto, à capacidade de retenção de água da carne, de modo que quanto maior o conteúdo de água fixada no músculo, maior a maciez da carne (ANADÓN, 2002). A textura da carne é determinada através de sua força de cisalhamento (BRESSAN, 1998).

\section{Transformação do músculo em carne}

Segundo SAMS (1999), embora o animal morra em questão de minutos após a sangria, suas células continuam a metabolizar e a responder por horas após a cessão da respiração. Durante este período, as células musculares continuam a utilizar a respiração aeróbica para produzir e consumir ATP (adenosina trifosfato). Quando acaba o oxigênio celular, a célula passa a depender apenas do metabolismo anaeróbico (glicólise) para o atendimento de suas necessidades de ATP (LAWRIE, 1991), utilizando-se das reservas de glicogênio muscular(VIEIRA, 1999). Assim, o músculo mantém a capacidade de contrair e relaxar (VIEIRA, 1999). De acordo com SAMS (1999), o glicogênio é convertido em ácido lático, produto final do metabolismo anaeróbico, que se acumula devido à falta de fluxo sanguíneo para removê-lo. Desta forma, a glicólise é inibida e a produção de ATP cessa. O músculo passa, então, a perder a capacidade de relaxamento, ficando em permanente contração entre actina e miosina (complexo actomiosina), no que se chama de rigor mortis propriamente dito, até que outros processos enzimáticos sejam iniciados. Segundo DRANSFIELD \& SOSNICKI (1999), a instalação do rigor mortis em frangos leva cerca de 1 hora, entretanto a velocidade de queda de $\mathrm{pH}$ pode variar entre linhagens e indivíduos. Tipicamente, valores de $\mathrm{pH}$ aferidos em 15 minutos após o abate variam de 6,2 a 6,6 em aves (DRANSFIELD \& SOSNICKI, 1999).

Após a instalação do rigor mortis, ocorre a degradação de proteínas responsáveis pela estrutura miofibrilar por um sistema enzimático proteolítico dependente de cálcio, composto pela enzima calpaína e seu inibidor calpastatina, liberando a tensão muscular e levando ao aumento da maciez da carne (VIEIRA, 1999).

Carne pálida, flácida e exsudativa, ou PSE

Um dos maiores problemas enfrentados pela indústria processadora é a questão da carne PSE, cujo termo tem origem nas iniciais das palavras inglesas pale, soft e exsudative, que significam carne pálida, flácida e exsudativa (DIRINCK et al., 1996). De acordo com LE BIHAN-DUVAL et al. (2003), os mecanismos fundamentais deste fenômeno ainda não foram bem elucidados em frangos. A carne PSE apresenta as propriedades funcionais comprometidas face à rápida glicólise post mortem, a qual acelera a queda de $\mathrm{pH}$ muscular enquanto a temperatura da carcaça ainda está alta (BARBUT, 1997; FERNANDEZ et al., 2002), levando à desnaturação de proteínas musculares (MOLETTE et al., 2003). Segundo DRANSFIELD \& SOSNICKI (1999), com relação à temperatura da carcaça durante o declínio de $\mathrm{pH}$, um aumento de $10^{\circ} \mathrm{C}$ aumenta a desnaturação de proteínas musculares em 20 vezes.

Segundo ANADÓN (2002), a dispersão de luz de uma superfície muscular é diretamente proporcional à sua quantidade de desnaturação protéica, o que, segundo LAWRIE (1991) e LE BIHANDUVAL et al. (2003), interfere na aparência física da carne, influenciando a quantidade de luz que lhe é refletida. Segundo OLIVO et al. (2001), quanto maior o grau de desnaturação protéica, menos luz é transmitida 
através das fibras e mais luz acaba sendo dispersa, o que leva à palidez da carne. Segundo OFFER \& KNIGHT (1988), o pH também influencia a capacidade de retenção de água da carne, pois o declínio de $\mathrm{pH}$ post mortem altera a composição celular e extracelular das fibras musculares, resultando em redução de grupos reativos disponíveis para reter água nas proteínas (OFFER \& KNIGHT, 1988). Segundo FLETCHER (1999), a ocorrência de PSE está relacionada com acúmulo excessivo de líquido nos produtos embalados, devido à sua menor capacidade de retenção de água, o que diminui a aceitação do produto pelo consumidor. O comprometimento das propriedades funcionais da carne PSE pode resultar em produtos industrializados de pouco rendimento (LARA et al., 2002), devido à liberação de exsudato, o que interfere na padronização durante a industrialização (BARBUT, 1997). Desta forma, segundo FERNANDEZ et al. (2002), o rendimento após o processamento da carne é altamente relacionado com a velocidade da queda de $\mathrm{pH}$ post mortem, de modo que uma diferença de uma unidade a menos no $\mathrm{pH}$ aferido aos 20 minutos post mortem corresponde a cerca de $2 \%$ a menos no rendimento após o processamento da carne. A capacidade de retenção de água da carne, por sua vez, exerce grande influência na maciez da carne (BRESSAN, 1998), já que na carne PSE a capacidade de retenção de água é menor, e quanto menor a quantidade de água no músculo, menor a maciez da carne (ANADÓN, 2002). De acordo com DRANSFIELD \& SOSNICKI (1999), a carne PSE possui também menor potencial proteolítico post mortem, o que contribui para a diminuição da maciez da carne. Segundo estes autores, o rápido declínio de $\mathrm{pH}$, a altas temperaturas da carcaça, inativa o sistema calpaína e reduz o amaciamento post mortem da carne.

O fenômeno PSE, portanto, é prognosticado pela combinação de análises de $\mathrm{pH}$, cor e capacidade de retenção de água nos músculos do peito (SWATLAND, 1995). De acordo com LARA et al. (2002), o fenômeno PSE em frangos pode ser detectado pela combinação dos valores de $\mathrm{pH}$ (abaixo de 5,8) e cor (valor L* acima de 52,0) aferidos em 24 horas após o abate. Fatores como estresse (OLIVO, 1999), tipo de atordoamento (SAMS, 1999) e temperatura de resfriamento (DUNN et al., 1995; VIEIRA, 1999) podem levar ao desencadeamento da condição PSE em aves.

Efeitos da seleção para características de desempenho, carcaça e composição corporal na qualidade da carne

Segundo DRANSFIELD \& SOSNICKI (1999) os problemas que vêm ocorrendo com a qualidade da carne de aves estão relacionados com a seleção destes animais para rápido crescimento, entretanto, os resultados encontrados na literatura muitas vezes divergem a este respeito. LE BIHANDUVAL et al. (1999) sugeriram uma relação entre a seleção para as características de desempenho que vem sendo empregada e a qualidade da carne em frangos. Estes autores utilizaram aves de linhagem selecionada para o peso vivo e o rendimento de peito e as compararam com aves de linhagem não-selecionada. A capacidade de retenção de água foi maior em aves selecionadas para maior peso de peito, mas a seleção para o peso vivo e para o peso de peito resultou em maior palidez da carne. Segundo DEBUT et al. (2003), aves selecionadas para alta taxa de crescimento tiveram maior velocidade de queda de $\mathrm{pH}$ post mortem e carne mais pálida, contudo a carne destas aves apresentou menores perdas de água por exsudação e maiores rendimentos após o processamento. Segundo FERNANDEZ et al. (2001), nem a velocidade nem a amplitude da queda de $\mathrm{pH}$ post mortem foram afetadas pela seleção para alta taxa de crescimento. Entretanto, de acordo com estes autores, em 24 horas post mortem, aves selecionadas para alta taxa de crescimento apresentaram carne mais pálida, mas menores perdas de água por exsudação e por cozimento e menor força de cisalhamento. De acordo com BERRI et al. (2001), a seleção para maiores peso vivo e rendimento de peito e menor deposição de gordura abdominal resultou em maior teor de proteína, e menores teores de umidade e mioglobina no músculo do peito dos frangos. Segundo estes autores, aves selecionadas exibiram menores perdas de água por exsudação e apresentaram menor velocidade e amplitude de queda de $\mathrm{pH}$ post mortem. Entretanto, a carne do peito destas aves foi mais pálida, com menor teor de vermelho e de amarelo do que aves não-selecionadas, condizendo com os menores teores de mioglobina encontrados no músculo destes animais. Segundo SANTÉ et al. (1995), em perus selecionados para alta taxa de crescimento, a velocidade de declínio de $\mathrm{pH}$ foi cerca de 0,04 unidade por minuto, duas vezes a velocidade de aves não selecionadas. Entretanto, de acordo com RÉMIGNON et al. (1996), não foram observadas diferenças na qualidade em termos de $\mathrm{pH}$, cor e perdas de água por exsudação em aves selecionadas a favor do peso do peito, quando comparadas com aves não-selecionadas.

As características das fibras musculares podem ser alteradas devido à seleção dos animais para alta taxa de crescimento, pois, segundo RÉMIGNON et al. (1995), aves selecionadas para alta taxa de crescimento tendem a possuir fibras musculares maiores e em maior número do que aves não selecionadas, entretanto a proporção de tipos de fibras musculares não se alteram entre estes animais. Segundo 
DRANSFIELD \& SOSNICKI (1999), a seleção para maiores taxas de crescimento pode resultar em maior diâmetro das fibras musculares.

Neste contexto, o estudo das correlações genéticas entre as características de qualidade de carne e as características normalmente utilizadas como critério nos programas de seleção de aves torna-se fundamental para a identificação das características relacionadas geneticamente à qualidade da carne e à condição PSE.

$\mathrm{O}$ pH aferido em 15 minutos após a evisceração possuiu baixas estimativas de correlações genéticas com o peso vivo e o rendimento de peito de frangos segundo LE BIHAN-DUVAL et al. (2001), que obtiveram estimativas que variaram entre $-0,06 \pm 0,02 \mathrm{e}$ $0,12 \pm 0,03$ entre estas características. Da mesma forma, o pH aferido em 24 horas após a evisceração não pareceu estar relacionado geneticamente com o peso vivo, o peso de peito e o rendimento de peito em frangos, uma vez que as estimativas de correlações genéticas entre estas características descritas por LE BIHAN-DUVAL et al. (1999; 2001) estiveram entre $0,12 \pm 0,04$ e $0,13 \pm 0,03$. Entretanto, de acordo com LE BIHAN-DUVAL et al. (2003), em perus, foi encontrada uma associação genética entre os pHs aferidos em 20 minutos e em 24 horas após a evisceração e o peso vivo e o rendimento de peito, tendo em vista as estimativas de correlações genéticas entre estas características encontradas por estes autores, que variaram entre $0,23 \pm 0,14$ e $0,62 \pm 0,10$. Assim, a seleção a favor do peso vivo, do peso de peito e do rendimento de peito pouco poderia interferir nos valores de $\mathrm{pH}$, de acordo com LE BIHAN-DUVAL et al. (1999; 2001). Todavia, segundo LE BIHAN-DUVAL et al. (2003), a seleção para estas características poderia incrementar estes valores.

A luminosidade foi diretamente correlacionada com o peso vivo e o peso de peito de frangos segundo LE BIHAN-DUVAL et al. (1999), tendo sido obtidas por estes autores estimativas de correlações genéticas de $0,51 \pm 0,08$ e $0,37 \pm 0,05$ entre estas características, respectivamente. Todavia, a luminosidade não foi associada geneticamente com o rendimento de peito, tendo em vista que a estimativa de correlação genética entre estas características encontrada pelos mesmos autores foi próxima de zero. Já de acordo com LE BIHAN-DUVAL et al. (2001), a luminosidade foi pouco correlacionada com o peso vivo e o rendimento de peito de frangos, com base nas estimativas de correlações genéticas obtidas por estes autores entre estas características, que foram de $0,16 \pm 0,01$ e $-0,07 \pm 0,03$, respectivamente. Entretanto, segundo LE BIHAN-DUVAL et al. (2003), a luminosidade da carne em perus pareceu ser associada geneticamente e inversamente com o peso vivo e o rendimento de peito, uma vez que as estimativas de correlações genéticas entre estas características foram de $-0,24 \pm 0,17$ e $-0,41 \pm 0,19$, respectivamente, de acordo com estes autores. Segundo LE BIHAN-DUVAL et al. (1999; 2001), o teor de vermelho pareceu estar relacionado geneticamente e inversamente com o peso vivo, o peso de peito e o rendimento de peito de frangos, tendo em vista as estimativas de correlações genéticas entre estas características encontradas por estes autores, que variaram entre $-0,51 \pm 0,04 \mathrm{e}-0,25 \pm 0,05$. Contudo, de acordo com LE BIHAN-DUVAL et al. (2003), o teor de vermelho em perus possuiu estimativas de correlações genéticas com o peso vivo e o rendimento de peito de $0,13 \pm 0,11$ e $-0,18 \pm 0,12$, respectivamente, não parecendo estarem estas características muito relacionadas geneticamente. Segundo os estudos encontrados na literatura, o teor de amarelo tende a apresentar uma associação genética inversa com o peso vivo, o peso de peito e o rendimento de peito das aves, tendo sido descritas entre estas características estimativas de correlações genéticas variando entre $-0,49 \pm 0,15$ e $-0,13 \pm 0,05$ (LE BIHANDUVAL et al., 1999; 2001; 2003), exceto por um dos resultados do estudo de LE BIHAN-DUVAL et al. (1999), que encontraram uma estimativa de correlação genética positiva e baixa entre o teor de amarelo e o peso vivo dos frangos, com valor de $0,11 \pm 0,06$. Desta forma, a seleção a favor do peso vivo, do peso de peito e do rendimento de peito parece ser capaz de aumentar a palidez da carne, através do aumento da luminosidade, segundo LE BIHAN-DUVAL et al. (1999), da redução dos teores de vermelho, segundo LE BIHAN-DUVAL et al. (1999; 2001) e dos teores de amarelo, segundo LE BIHAN-DUVAL et al. (1999; 2001; 2003). Entretanto, segundo LE BIHAN-DUVAL et al. (2001), a seleção a favor do peso vivo, do peso de peito e do rendimento de peito não teria efeito na luminosidade da carne, e de acordo com LE BIHAN-DUVAL et al. (2003), tal seleção poderia, inclusive, reduzir a luminosidade da carne.

As perdas de água por exsudação da carne foram pouco correlacionadas com o peso vivo e o rendimento de peito de frangos de acordo com LE BIHAN-DUVAL et al. (2001), tendo sido obtidas por estes autores estimativas de correlações genéticas de $-0,04 \pm 0,05$ e $-0,16 \pm 0,04$ entre estas características, respectivamente. Assim, a seleção a favor destas características de desempenho e carcaça pouco poderia interferir nas perdas de água por exsudação da carne.

Não pareceu existir associação genética entre o pH aferido em 15 minutos após a evisceração e o peso da gordura abdominal em frangos segundo LE 
BIHAN-DUVAL et al. (2001), uma vez que estes autores encontraram estimativa de correlação genética próxima de zero entre estas características. Entre o peso da gordura abdominal e o pH aferido em 24 horas após a evisceração, as estimativas de correlações genéticas encontradas por LE BIHAN-DUVAL et al. (1999; 2001) foram de $-0,64$ e $-0,54 \pm 0,04$, respectivamente. Desta forma, segundo estes autores, a seleção contra a deposição de gordura abdominal parece ser capaz de incrementar o pH aferido em 24 horas após a evisceração. No estudo de LE BIHAN-DUVAL et al. (1999), a deposição de gordura abdominal foi associada geneticamente com a luminosidade e os teores de vermelho e amarelo, tendo em vista as estimativas de correlações genéticas entre estas características encontradas por estes autores, que foram de $0,48 \pm 0,16$, $0,13 \pm 0,05$ e $0,36 \pm 0,11$, respectivamente. Ou seja, segundo estes autores, a seleção contra a deposição de gordura abdominal poderia diminuir a luminosidade da carne. Segundo LE BIHAN-DUVAL et al. (2001), a luminosidade e também as perdas de água por exsudação foram associadas geneticamente com a deposição de gordura abdominal em frangos, tendo sido obtidas por estes autores estimativas de

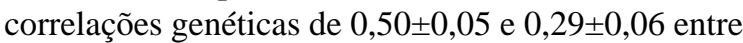
estas características, respectivamente. Segundo os mesmos autores, as estimativas de correlações genéticas entre o peso da gordura abdominal e os teores de vermelho e amarelo foram de $-0,24 \pm 0,03 \mathrm{e}$ $0,02 \pm 0,04$, respectivamente. Estes autores sugeriram que a seleção contra a deposição de gordura abdominal nos frangos pode contribuir para reduzir a luminosidade da carne e também as perdas de água por exsudação.

Estimativas de herdabilidades para as características de qualidade de carne

A genética possui grande impacto nos processos bioquímicos durante o rigor mortis e no desenvolvimento do fenômeno PSE em frangos (SAMS, 1999). Segundo LE BIHAN-DUVAL et al. (2003), a qualidade da carne das aves é influenciada por um grande número de fatores, mas principalmente pela genética, uma vez que níveis significantes de herdabilidade foram obtidos para várias características de qualidade em recentes estudos.

A estimativa de herdabilidade para o $\mathrm{pH}$ aferido em 15 minutos após a evisceração em frangos foi de 0,49 $\pm 0,01$ de acordo com LE BIHAN-DUVAL et al. (2001). Segundo LE BIHAN-DUVAL et al. (2003), a estimativa de herdabilidade do $\mathrm{pH}$ aferido em 20 minutos após a evisceração em perus foi de $0,21 \pm 0,04$. As estimativas de herdabilidade encontradas por LE
BIHAN-DUVAL et al. (1999; 2001; 2003) em aves para o $\mathrm{pH}$ aferido em 24 horas após a evisceração foram de $0,49 \pm 0,11,0,35 \pm 0,03$ e $0,16 \pm 0,04$, respectivamente. Assim, segundo estes estudos, as medidas de $\mathrm{pH}$ nos tempos estudados, de modo geral, parecem ser capazes de apresentar uma boa resposta à seleção.

Em relação aos parâmetros de cor da carne de aves, LE BIHAN-DUVAL et al. (1999; 2001; 2003) encontraram estimativas de herdabilidade de $0,75 \pm 0,08$, $0,50 \pm 0,03$ e 0,12 $\pm 0,04$ para a luminosidade, de 0,81 $\pm 0,04$, $0,57 \pm 0,02$ e $0,21 \pm 0,05$ para o teor de vermelho e de $0,64 \pm 0,06,0,55 \pm 0,04$ e $0,14 \pm 0,04$ para o teor de amarelo, respectivamente. Segundo LE BIHAN-DUVAL et al. (2001), a estimativa de herdabilidade das perdas de água por exsudação da carne de frangos foi de $0,39 \pm 0,04$. Desta forma, parece haver um predominante papel da genética no controle da cor e da capacidade de retenção de água da carne, indicando que estas características podem apresentar uma satisfatória resposta à seleção.

Estimativas de correlações genéticas entre as características de qualidade de carne

De acordo com SWATLAND (1995), podese prognosticar a condição PSE pela combinação dos resultados de $\mathrm{pH}$, cor e capacidade de retenção de água da carne, tornando-se importante, desta forma, o estudo das correlações genéticas entre estas características para uma melhor caracterização deste fenômeno.

$\mathrm{O}$ pH aferido em 15 minutos após a evisceração foi pouco correlacionado geneticamente com a luminosidade segundo LE BIHAN-DUVAL et al. (2001), tendo sido estimada correlação genética entre estas características de $0,13 \pm 0,03$ por estes autores em frangos. Já LE BIHAN-DUVAL et al. (2003) encontraram estimativa de correlação genética inversa entre o $\mathrm{pH}$ aferido em 20 minutos após a evisceração e a luminosidade da carne de perus, com valor de $-0,80 \pm 0,10$, o que, segundo estes autores, é um indicativo da condição PSE. Segundo LE BIHAN-DUVAL et al. (1999; 2001; 2003), pareceu existir uma associação genética inversa entre o pH aferido em 24 horas após a evisceração e a luminosidade da carne das aves, tendo sido obtidas por estes autores estimativas de correlações genéticas de $-0,65 \pm 0,10,-0,91 \pm 0,02$ e $0,53 \pm 0,19$, respectivamente, entre estas características.

Os pHs aferidos em 15 e 20 minutos após a evisceração foram correlacionados geneticamente com o teor de vermelho da carne das aves, segundo LE BIHAN-DUVAL et al. (2001; 2003), tendo sido estimadas por estes autores correlações genéticas entre estas características de $-0,23 \pm 0,03$ e $-0,25 \pm 0,11$, respectivamente. De acordo com LE BIHAN-DUVAL 
et al. (1999; 2001) o pH aferido em 24 horas após a evisceração foi pouco associado geneticamente com o teor de vermelho da carne de frangos, tendo em vista as estimativas de correlações genéticas encontradas por estes autores entre estas características, de $0,11 \pm 0,08$ e $0,14 \pm 0,06$, respectivamente.

Segundo LE BIHAN-DUVAL et al. (2001) o pH aferido em 15 minutos após a evisceração foi pouco correlacionado geneticamente com o teor de amarelo da carne de frangos, tendo sido estimada correlação genética entre estas características próxima de zero por estes autores. Entretanto, de acordo com LE BIHANDUVAL et al. (2003), o teor de amarelo esteve geneticamente e inversamente relacionado com o $\mathrm{pH}$ aferido em 20 minutos após a evisceração, com base na estimativa de correlação genética entre estas características obtida por estes autores, que foi de $0,35 \pm 0,20$. Da mesma forma, segundo LE BIHANDUVAL et al. (2001), a estimativa de correlação genética entre o pH aferido em 24 horas após a evisceração e o teor de amarelo na carne de frangos foi de $-0,43 \pm 0,04$. Contudo, de acordo com LE BIHAN-DUVAL et al. (1999), o pH aferido em 24 horas após a evisceração foi pouco associado geneticamente com o teor de amarelo da carne de frangos, tendo em vista a estimativa de correlação genética encontrada por estes autores entre estas características, de $-0,11 \pm 0,11$.

Com base na maior parte destes estudos, portanto, pode-se concluir que carnes com maior luminosidade, de modo geral, estão associadas a baixos pHs. Da mesma forma, baixos pHs parecem ser associados, de modo geral, com maiores teores de vermelho e amarelo.

A estimativa de correlação genética entre o pH aferido em 15 minutos após a evisceração e as perdas de água por exsudação da carne de frangos foi de $-0,29 \pm 0,04$ de acordo com LE BIHAN-DUVAL et al. (2001). Desta forma, segundo este estudo, quanto mais ácida a carne, maiores suas perdas de água por exsudação. LE BIHAN-DUVAL et al. (2001) encontraram alta correlação genética entre o pH aferido em 24 horas após a evisceração e as perdas de água por exsudação da carne de frangos, tendo obtido estimativa de correlação

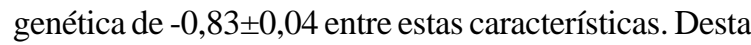
forma, estes autores sugeriram que a seleção para o pH aferido em 24 horas após a evisceração poderia ser útil em prevenir carnes excessivamente exsudativas.

Maiores perdas de água por exsudação pareceram estar relacionadas geneticamente com uma maior luminosidade da carne de frangos, com base na estimativa de correlação genética obtida entre estas características por LE BIHAN-DUVAL et al. (2001), que foi de $0,80 \pm 0,03$. Segundo estes autores, a seleção para menor luminosidade poderia diminuir as perdas de água por exsudação da carne. De acordo com este estudo, a luminosidade pareceu estar relacionada às perdas de água por exsudação da carne não apenas indiretamente, por meio de sua alta correlação com o pH aferido em 24 horas após a evisceração, o qual, por sua vez, é correlacionado com estas perdas, conforme já mencionado. Segundo este estudo, a luminosidade pareceu relacionar-se com as perdas de água por exsudação também diretamente, motivo pelo qual estes autores indicaram a seleção contra a luminosidade como critério para reduzir as perdas de água por exsudação, uma vez que esta característica pareceu ser bastante herdável, segundo LE BIHAN-DUVAL et al. (1999; 2001). As perdas de água por exsudação pareceram ser inversamente relacionadas com o teor de vermelho e pouco associadas com o teor de amarelo da carne de frangos, com base nas estimativas de correlações genéticas obtidas entre estas características por LE BIHAN-DUVAL et al. (2001), que foram de $-0,25 \pm 0,07$ e $0,16 \pm 0,07$, respectivamente.

De acordo com LE BIHAN-DUVAL et al. (2001) os pHs aferidos em 15 minutos e em 24 horas após a evisceração em frangos parecem ser controlados por genes diferentes, pois neste estudo foi estimada uma correlação genética próxima de zero entre estas características. Segundo SOSNICKI et al. (1998) estes parâmetros são controlados por mecanismos distintos: o pH aferido em 24 horas após a evisceração é principalmente determinado pela reservas iniciais de glicogênio do músculo ao abate, enquanto o $\mathrm{pH}$ aferido logo após a evisceração é mais dependente da atividade das enzimas glicolíticas post mortem. De acordo com LARZUL et al. (1999), em suínos, a reserva de glicogênio muscular foi geneticamente relacionada com o pH aferido em 24 horas após a evisceração, de modo que maiores pHs estariam relacionados com menores reservas de glicogênio muscular. Entretanto, segundo estes autores, a reserva de glicogênio não se apresentou relacionada com o $\mathrm{pH}$ aferido em 30 minutos após a evisceração. Por outro lado, segundo LE BIHAN-DUVAL et al. (2003) os pHs aferidos em 15 minutos e em 24 horas após a evisceração foram geneticamente correlacionados, tendo sido obtida estimativa correlação genética de $0,59 \pm 0,10$ entre estas características por estes autores em perus.

As estimativas de correlações genéticas entre a luminosidade e o teor de vermelho da carne de frangos foram de $-0,45 \pm 0,05$ e $-0,48 \pm 0,05$ segundo LE BIHAN-DUVAL et al. (1999; 2001), respectivamente, parecendo haver desta forma um antagonismo entre estas características. Contudo, de acordo com LE BIHAN-DUVAL et al. (2003), a estimativa de correlação 
genética entre a luminosidade e o teor de vermelho da carne de perus foi de $0,12 \pm 0,04$, não parecendo haver uma associação genética importante entre estas características. Segundo LE BIHAN-DUVAL et al. (1999) a luminosidade não foi correlacionada geneticamente com o teor de amarelo em frangos, tendo sido obtida uma estimativa de correlação genética próxima de zero entre estas características por estes autores. Entretanto, de acordo com LE BIHAN-DUVAL et al. $(2001 ; 2003)$ as estimativas de correlações genéticas entre a luminosidade e o teor de amarelo foram de 0,20 $\pm 0,04$ e 0,54 $\pm 0,18$, respectivamente, indicando haver uma associação genética entre estas características em aves. De acordo com LE BIHANDUVAL et al. (1999; 2001) as estimativas de correlações genéticas entre os teores de vermelho e amarelo em

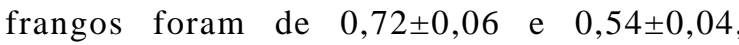
respectivamente, indicando que estas características estariam associadas geneticamente. Todavia, segundo com LE BIHAN-DUVAL et al. (2003) a estimativa de correlação genética entre estas características em perus foi próxima de zero. A maior parte destes estudos foi indicativa de que carnes com maior luminosidade tendem a apresentar menores teores de vermelho e maiores teores de amarelo, e carnes com maiores teores de vermelho tendem a apresentar maiores teores de amarelo.

\section{CONCLUSÃO}

Apesar dos prejuízos da indústria devido a problemas com a qualidade da carne, pouca atenção vem sendo dada ao problema PSE, especialmente em frangos. O estudo dos parâmetros genéticos das características relacionadas à condição PSE pode favorecer a obtenção de produtos de melhor qualidade sensorial e de maior rentabilidade, fazendo-se necessário um monitoramento dos atributos de qualidade da carne e até mesmo sua inclusão nos índices de seleção em um programa de melhoramento genético. Além disso, deve-se identificar as características associadas à qualidade da carne e também analisar os atributos de qualidade em genótipos utilizados comercialmente, para que se obtenham informações que levem à redução da condição PSE nestas linhagens.

\section{REFERÊNCIAS}

ANADÓN, H. L. S. Biological, nutritional and processing factors affecting breast meat quality of broilers. 2002. 171f. Thesis (Doctor of Philosophy in Animal and Poultry Sciences) - Faculty of Virginia Polytechnic Institute and State University.
BARBUT, S. Problem of pale soft exsudative meat in broiler chickens. British Poultry Science, Roslin, v. 38, p. 355358, 1997

BERRI, C. et al. Effect of selection for improved body composition on muscle and meat characteristics of broilers from experimental and commercial lines. Poultry Science, Savoy, v. 80, p. 833-838, 2001.

BRESSAN, M. C. Efeito dos fatores pré e pós-abate sobre a qualidade da carne de peito de frango. 1998. 201p. Tese (Doutorado em Tecnologia de Alimentos) - Faculdade de Engenharia de Alimentos, UNICAMP.

CAMPOS, E. J; PEREIRA, J. C. C. Melhoramento genético das aves. In: PEREIRA, J. C. C. Melhoramento genético aplicado à produção animal. Belo Horizonte : FEP-MVZ, 1999. Cap. 17, p. 284-314.

DEBUT, M. et al.Variation of chicken technological meat quality in relation to genotype and preslaughter stress conditions. Poultry Science, Savoy, v. 82, p. 1829-1838, 2003.

DIRINCK, P. et al. Studies on vitamin E and meat quality. 1. Effect of feeding high vitamin E levels on time-related pork quality. Journal of Agricultural Food and Chemistry, Columbus, v. 44, p. 65-68, 1996.

DRANSFIELD, E.; SOSNICKI, A. A. Relationship between muscle growth and poultry meat quality. Poultry Science, Savoy, v. 78, p. 743-746, 1999.

DUNN, A. A. et al. Contribution of "rigor shortening" and "cold shortening" to variability in the texture of pectoralis major muscle from commercially processed broilers. British Poultry Science, Roslin, v. 36, n.4, p. 401-413, 1995.

FENNEMA, O. R. Comparative water holding properties of various muscle food. Journal of Muscle Foods, Urbana, n. 1, p. $363-381,1990$.

FERNANDEZ, X. et al. Post mortem muscle metabolism and meat quality in three genetic types of turkey. British Poultry Science, Roslin, v. 42, p. 462-469, 2001.

FERNANDEZ, X. et al. Effects of the rate of muscle post mortem $\mathrm{pH}$ fall on the technological quality of turkey meat. British Poultry Science, Roslin, v. 43, p. 245-252, 2002.

FLETCHER, D. L. Broiler breast meat color variation, $\mathrm{pH}$ and texture. Poultry Science, Savoy, v. 78, p. 1323-1327, 1999.

LARA, J. A. F. et al. Estresse térmico e incidência de carne PSE em frangos. Revista Brasileira de Ciência Avícola, Campinas, n. 4, p.15, 2002.

LARZUL, C. P. et al. Selection for reduced muscle glycolitic potential in Large White pigs. II. Correlated responses in meat quality and muscle compositional traits. Genetics, Selection, Evolution, Jouy-en-Josas, v.31, p. 61-76, 1999.

LAWRIE, R. A. Meat science. New York : Pergamon, 1991. 293 p.

LE BIHAN-DUVAL, E. et al. Broiler meat quality: effect of selection for increased carcass quality and estimates of genetic parameters. Poultry Science, Savoy, v. 78, p. 822-826, 1999. 
LE BIHAN-DUVAL, E. et al. Estimation of the genetic parameters of meat characteristics and their genetic correlations with grow and body composition in a experimental broiler line. Poultry Science, Savoy, v. 80, n. 7, p. 839-843, 2001

LE BIHAN-DUVAL, E. et al. Genetic parameters of meat technological quality traits in a grand-parental commercial line of turkey. Genetics, Selection, Evolution, Jouy-en-Josas, v. 35, p. 623-635, 2003.

MOLETTE, C. et al. Effect of rate of $\mathrm{pH}$ fall on turkey breast meat quality. British Poultry Science, Roslin, v. 44, n. 5, p. 787-788, 2003.

OFFER, G.; KNIGHT, P. The structural basis of water-holding in meat. Part 1: General principles and water uptake in meat processing. In: LAWRIE, R. A. Developments in meat science. London : Elsevier, 1988. p. 63-171.

OLIVO, R. Carne PSE em frangos. 1999. 97f. Tese (Doutorado em Ciências dos Alimentos) - Faculdade de Ciências Farmacêuticas, Universidade de São Paulo.

OLIVO, R. et al. Fatores que influenciam na cor de filés de peito de frango. Revista Nacional da Carne, São Paulo, v. 25, n. 289 , p. $44-49,2001$.

PARK, G. B. et al. Influence of slaughter yield and quality grades of Hanwoo (Korean native cattle) carcasses. Journal of Animal Science, Savoy, v. 80, p. 129-136, 2002.

RÉMIGNON, H. et al. Selection for rapid growth increases the number and the size of muscle fibres without changing their typing in chickens. Journal of Muscle Research and Cell Motility, London, v. 16, p. 95-102, 1995.

RÉMIGNON, H. et al. Influence of increasing breast meat yield on muscle histology and meat quality in the chicken. Reproduction, Nutrition, Development, Jouy-en-Josas, v. 36, p. 523-530, 1996.

SAMS, A. R. Meat quality during processing. Poultry Science, Savoy, v. 78, p. 798-803, 1999.

SANTÉ, V. et al. Impact of turkey breeding and production on breast meat quality. In: EUROPEAN SYMPOSIUM ON THE QUALITY OF POULTRY MEAT, 12., 1995, Zaragoza. Proceedings... Zaragoza : Organising Committee $12^{\text {th }}$ European Symposium on the Quality of Poultry Meat, 1995. p. 151-156.

SOSNICKI, A. A. et al. PSE-like syndrome in breast muscle of domestic turkeys: a review. Journal of Muscle Foods, Urbana, v. 9, p. 13-23, 1998.

SWATLAND, H. J. On line evaluation of meat. Lancaster : Technomic, 1995. 343p.

VIEIRA, S. L. Considerações sobre as características de qualidade de carne de frango e fatores que podem afetála. Porto Alegre, 1999. Capturado em 17 out. 2004. Online. Disponível na Internet http://www.sbz.org.br/eventos/ PortoAlegre/homepagesbz/Sergio.htm. 